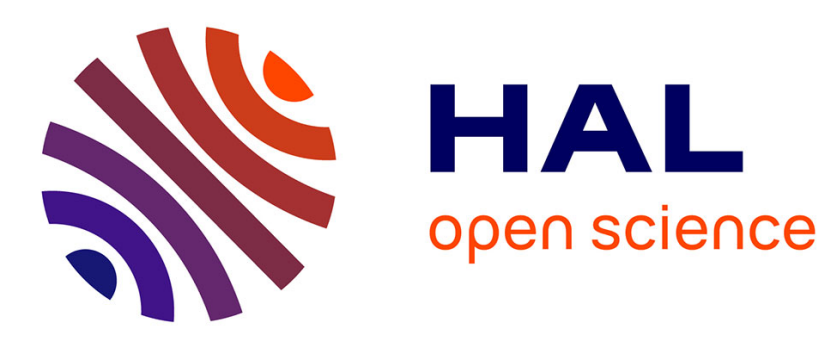

\title{
Experimental characterization of NiTi SMAs thermomechanical behaviour using temperature and strain full-field measurements
}

Pauline Schlosser, Denis Favier, Hervé Louche, Laurent Orgéas

\section{- To cite this version:}

Pauline Schlosser, Denis Favier, Hervé Louche, Laurent Orgéas. Experimental characterization of NiTi SMAs thermomechanical behaviour using temperature and strain full-field measurements. Advances in Science and Technology, 2008, 59, pp.140-149. 10.4028/3-908158-16-8.140 . hal-00336736

\section{HAL Id: hal-00336736 https://hal.science/hal-00336736}

Submitted on 27 Jan 2019

HAL is a multi-disciplinary open access archive for the deposit and dissemination of scientific research documents, whether they are published or not. The documents may come from teaching and research institutions in France or abroad, or from public or private research centers.
L'archive ouverte pluridisciplinaire HAL, est destinée au dépôt et à la diffusion de documents scientifiques de niveau recherche, publiés ou non, émanant des établissements d'enseignement et de recherche français ou étrangers, des laboratoires publics ou privés. 


\title{
Experimental characterization of NiTi SMAs thermomechanical behaviour using temperature and strain full-field measurements.
}

\author{
P. Schlosser ${ }^{1,2, a}$, D. Favier ${ }^{1, b}$, H. Louche ${ }^{2, c}$, L. Orgéas ${ }^{1, d}$ \\ ${ }^{1}$ Université de Grenoble / CNRS, Laboratoire 3S-R, BP 53, 38041, Grenoble cedex 9, France. \\ 2 Université de Savoie, SYMME, BP 74944 Annecy le Vieux Cedex, France. \\ apauline.schlosser@hmg.inpg.fr, ${ }^{b}$ denis.favier@hmg.inpg.fr, \\ cherve.louche@univ-savoie.fr, laurent.orgeas@hmg.inpg.fr
}

Keywords: NiTi, shape memory alloy, superelasticity, localisation, strain and temperature full field measurement, heat sources estimation

\begin{abstract}
.
The tension behaviour of initially austenitic NiTi thin wall tubes was investigated using measurements of temperature and strain fields simultaneously. The first specimen was totally superelastic but the unloading was performed before the end of the loading stress plateau. The second specimen loading was performed beyond the stress plateau to allow analyzing the unloading, but was not superelastic and at a faster strain rate. Both tests show homogeneous behaviour at the beginning of the loading. Strong localisations, taking the shape of helical bands, are observed during the loading and unloading stress plateaus. To obtain quantitative energy information, allowing a better recognition of the deformation mechanisms, an estimation of the local heat sources based on image processing of the temperature fields is presented. Two methods of heat sources estimation allowing analysis of deformation mechanisms are proposed in the present paper: first during the homogeneous, then during localized stages.
\end{abstract}

\section{Introduction}

Shape Memory Alloys (SMAs) are now employed in a large number of applications in the fields of aeronautical, biomedical and structural engineering. The applications based on the peculiar properties of these SMAs are more and more designed using numerical simulation and finite element softwares in which three dimensional constitutive equations are implemented to model the thermomechanical behaviour of these SMAs. These constitutive equations are based on uniaxial and multiaxial experiments within a wide temperature range assuming homogeneous deformation of the specimens. The aim of the present paper is to illustrate, on two examples, information that can be deduced from temperature and strain full field measurements performed during these tests. This information is fundamental for the establishment of more efficient model.

The present paper is focused on the superelastic behaviour of NiTi polycrystalline SMAs. Owing to their outstanding superelastic behaviour at human body temperature and to their biocompatibility, polycrystalline Ti-50.8\%at Ni SMAs are being increasingly used for biomedical applications (human implants and surgery instruments). Design of these applications uses constitutive equations mostly based on the conventional understanding of the superelastic tensile engineering strain-stress curve shown in Fig.1. Initially, the material is in austenite phase (A) since the testing temperature is above $A_{f} . A_{f}$ is the temperature at which the martensite phase (M) stable at low temperature is completely transformed by heating to the austenite phase A stable at high temperature. The loading curve of Fig. 1 is usually divided in a first linear part (1) associated with the elastic deformation of A, a second one (2) associated with the transformation A-M occurring along an upper stress plateau and then a third one (3) associated with the elastic deformation of 
oriented martensite variants. When the load is progressively removed, the reverse transformation M-A occurs along a lower stress plateau (5) after an initial linear stage (4) associated with the elastic unloading of the oriented martensite $\mathrm{M}$. The complete reverse transformation is followed by the elastic unloading of A during the stage (6). Modelling of the superelastic behaviour of NiTi SMAs based on this above simple analysis is a preliminary step. However, a better understanding of the complex phenomena occurring during all these stages is necessary to improve this modelling.



Figure 1: Oversimplified superelastic strain-stress curve. Conventional understanding: (1) Elastic deformation of austenite A. (2) Austenite to oriented martensite transformation A-M. (3) and (4) Elastic deformation of oriented martensite. (5) Oriented martensite to austenite reverse transformation M-A. (6) Elastic deformation of austenite A.

In that respect, within the past decades, intensive experimental investigations have been carried out to characterize and understand deformation mechanisms associated with the superelasticity of NiTi SMAs. Most of these studies have been achieved in tension using wires, strips or bone-shaped samples. It is now well-established that uniaxial tensile superelastic deformation of polycrystalline NiTi SMAs often exhibits localised Lüders-like deformation modes [1-9] which occur during the upper (2) and lower (5) plateaus sketched in Fig.1. This heterogeneous deformation may disappear for other mechanical loadings such as compression or shear [5] or tension-torsion combined tests [6], and can also be observed during the ferroelastic tensile deformation of polycrystalline NiTi SMAs [10]. The localisation has been studied using qualitative optical observations [1,3, 6-8], multiple extensometers [2, 4-5] and full-field temperature measurements [3, 9]. Comparatively, the other stages (1), (3), (4) and (6) have been less studied. However, Brinson et al [7] present some results showing clearly the occurrence of several deformation mechanisms other than elastic distortion of the crystalline structure during each of these three stages.

Studies in the references cited earlier and other numerous works have brought up precious issues in the fundamental understanding and modelling of stress-induced phase transformation in polycrystalline NiTi SMAs. For optimal implementation of NiTi SMAs in engineering applications, a thorough understanding of the material behavior is necessary. In that order, the present contribution reports and analyses results concerning tensile tests on NiTi polycrystalline thin walled tubes during which thermal as well as kinematical full-field measurements have been simultaneously carried out.

\section{Experimental}

Experimental set-up. A special designed gripping system (Fig. 2a) was created using a pair of standardized collets and collet holders (2) with a pin inside to avoid crushing the tube (1) [11]. Both collet holders are linked to an universal mechanical tensile testing machine (Instron 5569, 50kN) via two driving shafts (3) in order to avoid any bending of the tensile specimen. During the tests, the usual mechanical data (axial force $F$, crosshead displacement $U$ ) as well as the thermal and kinematical fields were acquired simultaneously with two digital cameras, respectively an infrared and a visible one (Fig. 2.a). 
The temperature field was obtained with a fast multidetector infrared camera (Cedip Jade III MW, $145 \mathrm{~Hz}$ ). The infrared camera has a resolution of $320 \times 240$ pixels. The spatial resolution (pixel size), that depends on the adjustment of the focal distance, was estimated to be close to $0.4 \mathrm{~mm}$ for the carried tests. Prior to the tests, the surface of the tube was coated by a highly emissive paint in order to obtain blackbody properties compatible with the calibration law of the camera, this leaded to an accuracy on temperature variations less than $0.1 \mathrm{~K}$.

The displacement field of any particle of the outer surface of the tube has been obtained using a digital visible camera (Hamamatsu, $1280 \times 1024$ pixels, $9 \mathrm{~Hz}$, ambient lighting) and a Digital Image Correlation (DIC) processing ("7D" software [12]). To obtain blackbody properties a black paint was spread on the tube. Moreover white paint speckles were superimposed to enhance DIC. The elementary cell of the correlation grid was $10 \times 10$ pixels, so the spatial resolution was estimated to be of the order of $0.5 \times 0.5 \mathrm{~mm}^{2}$, as shown in Fig. 2.c. With such parameters, the accuracy on the strain measurement was $<0.1 \%$.

The nominal stress $\sigma_{0}$ is obtained by dividing the measured force by the initial cross section of the tube. The nominal strain is obtained by dividing the difference of displacements $\Delta l$ of two points $\mathrm{A}$ and $\mathrm{B}$ initially distant of the gauge length $\mathrm{L}_{0}$ by this length (Fig.2.b).

(A)

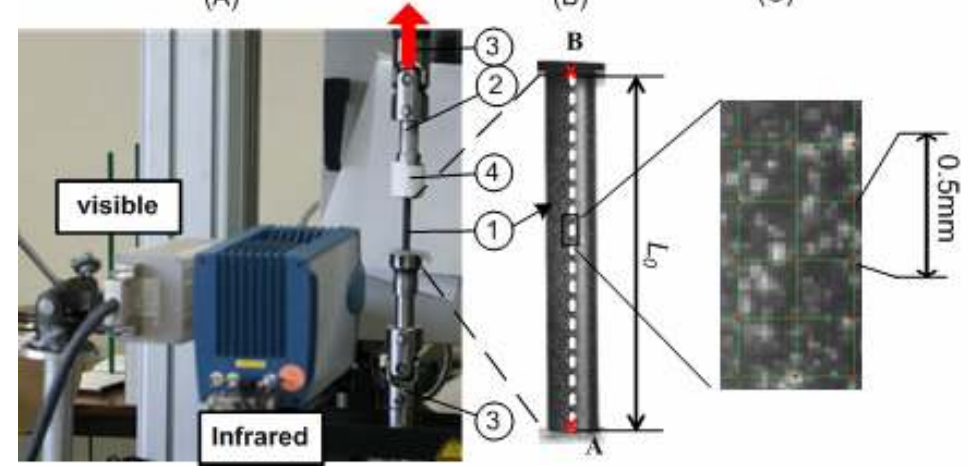

Figure $2:$ a) Experimental setup with the two infrared and visible digital cameras, b) view of the tube (1) in the centre, c) Close-up showing the virtual grid and the surface of the tube coated with a random pattern of white painted speckles superimposed on black paint.

Tensile specimens. Two tube samples were used. The first sample has a diameter of $10 \mathrm{~mm}$ and a thickness of $0.5 \mathrm{~mm}$ whereas the second sample has a diameter of $6 \mathrm{~mm}$ and a thickness of 0.1 $\mathrm{mm}$. The gauge lengths were respectively 71 and $82 \mathrm{~mm}$. Fig. 3 represents the DSC measurements of the two samples. Both DSC exhibit two-stage transformations austenite $(\mathrm{A}) \rightarrow \mathrm{R}$ phase $(\mathrm{R}) \rightarrow$ martensite $(\mathrm{M})$ during cooling and $\mathrm{M} \rightarrow \mathrm{R} \rightarrow \mathrm{A}$ during heating.

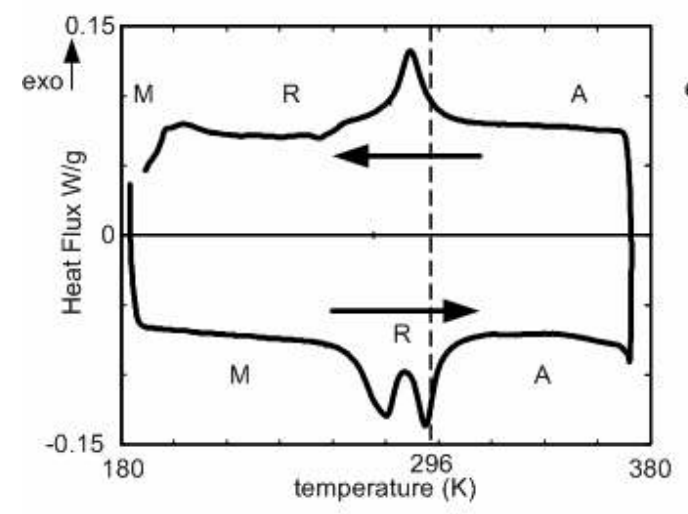

a)

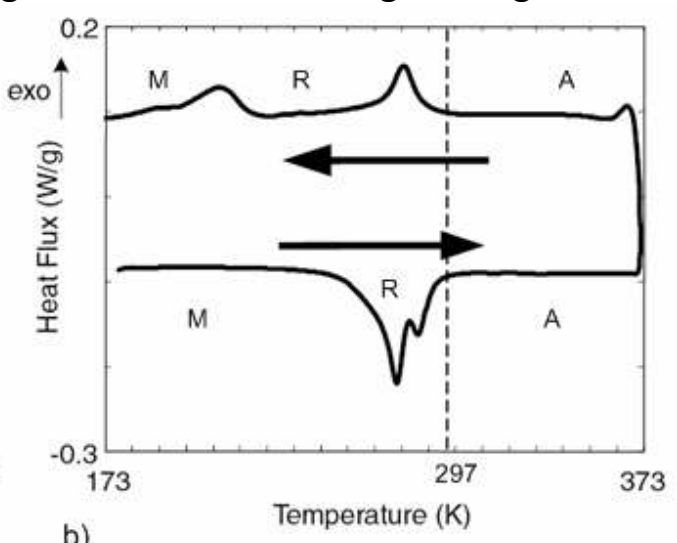

b)

Figure 3 : DSC measurements of a) the first sample, b) the second sample. The dash lines represent the tensile test room temperature $T_{a}$.

Tensile tests. The tensile tests were conducted in air at constant cross head velocity $\dot{\mathrm{U}}=1 \mathrm{~mm} / \mathrm{min}$ for specimen 1 and $\dot{\mathrm{U}}=5 \mathrm{~mm} / \mathrm{min}$ for specimen 2 . The nominal axial strain rates are 
respectively $\dot{\varepsilon}=\dot{U} / L_{0}=2.3510^{-4} \mathrm{~s}^{-1}$ and $\dot{\varepsilon}=10^{-3} \mathrm{~s}^{-1}$. Before being clamped, each tube was heated up to $373 \mathrm{~K}$ and cooled down to the ambient temperature $T_{a}$, plotted in dashed lines in Fig. 3 . The initial tube temperature $T_{0}$ is homogeneous and equal to ambient temperature $T_{a}$ so that the material was in the austenite state with a small amount of initial R-phase for sample 1 and almost totally austenitic for sample 2 .

\section{Results}

Macroscopic results. The curves plotted in Fig. 4.a and 4.b show the nominal axial stress $\sigma_{0}=F / S_{0}$ ( $S_{0}$ being the initial cross-section of the tube) as a function of the nominal axial strain $\varepsilon$ respectively on the first and the second sample. The nominal axial strain $\varepsilon$ is deduced from the displacements of the two extremities A and B obtained with DIC. Markers in the two curves show typical times.

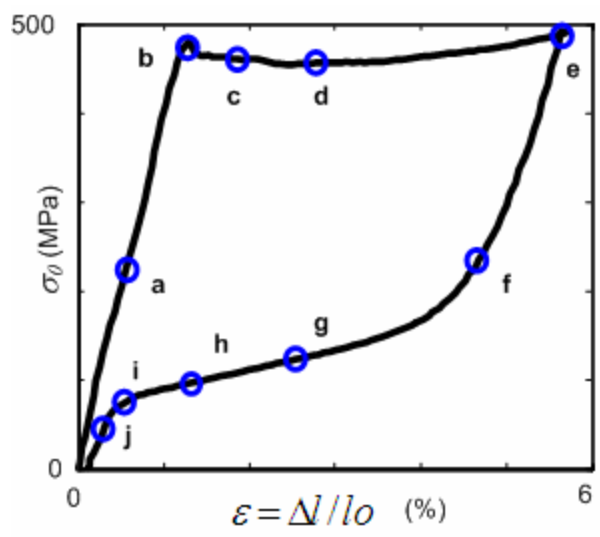

a)

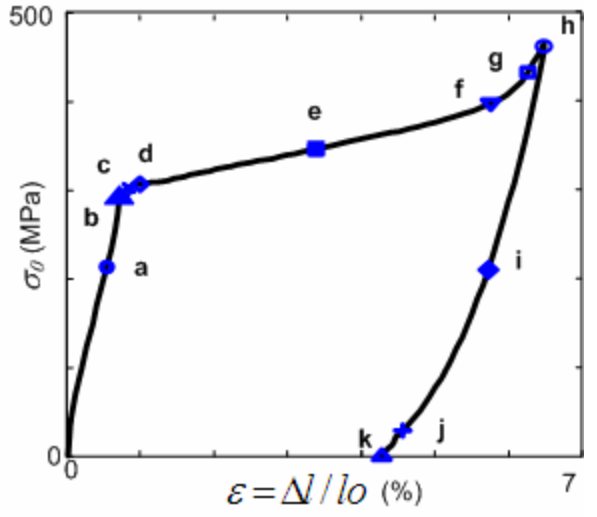

b)

Figure 4 : Nominal stress-strain curve obtained during tensile tests performed at room temperature: a) at $1 \mathrm{~mm} / \mathrm{min}$ on the first superelastic sample. b) at $5 \mathrm{~mm} / \mathrm{min}$ on the second sample initially austenitic, but not totally superelastic at the testing temperature.

Temperature and strain fields measurements. Fig. 5 represents the axial Green-Lagrange strain field $E_{x x}(\mathrm{M}, \mathrm{t})$ and the temperature variation field $\theta(\mathrm{M}, \mathrm{t})=T(\mathrm{M}, \mathrm{t})-T(\mathrm{M}, 0)$ on the first sample at characteristic times (a, c, e, f, h) of Fig.4.a. M design spatial points.

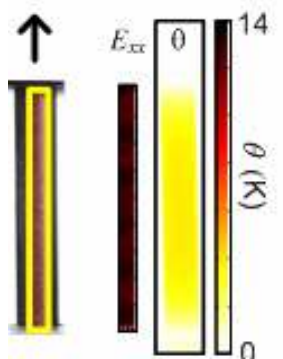

(1)

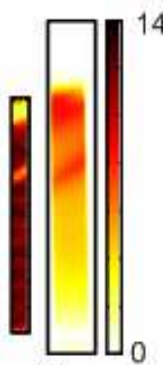

(c)

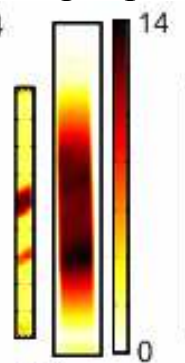

(e)

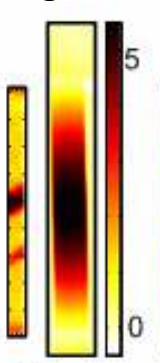

(f)

(2)

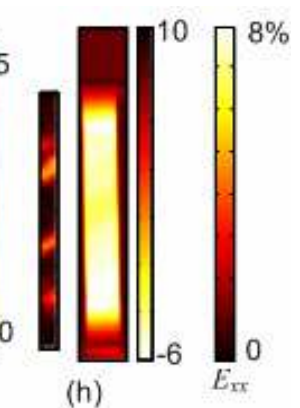

(h)

Figure 5: Tensile test on sample 1. (1) sample with in yellow the limit of the image correlation area.

(2 a to h) Coloured maps showing the local Green-Lagrange strain $E_{x x}(M, t)$ (left) and the temperature variation $\theta(M, t)$ (right) fields on the surface of the deformed tube at the five selected times (a,c,e,f,h) marked in Fig.4.a.

Fig.6 represents, for specimen 1, the evolution of the axial Green-Lagrange strain $E_{x x}(\mathrm{x}, \mathrm{y}=0, \mathrm{t})$ and the temperature variation $\theta(\mathrm{x}, \mathrm{y}=0, \mathrm{t})$ along one tube generating line $(\mathrm{y}=0)$ at the selected times marked in Figure 4.a. 
a)

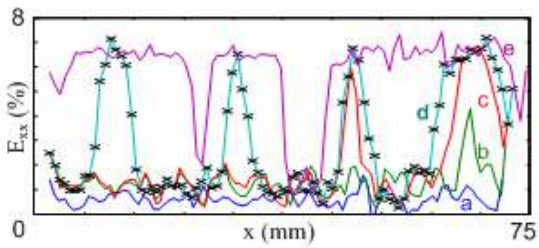

b)

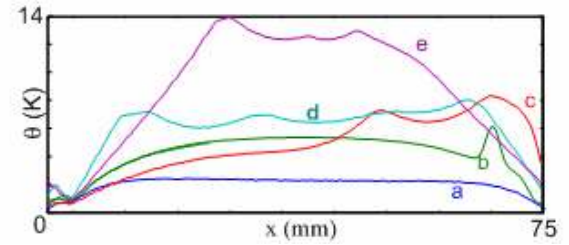

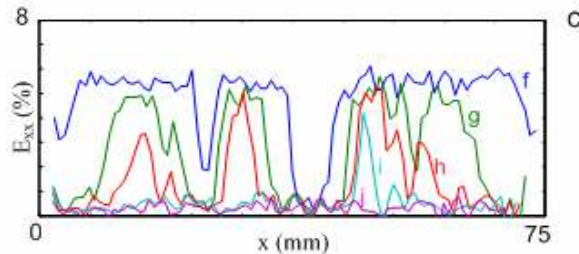

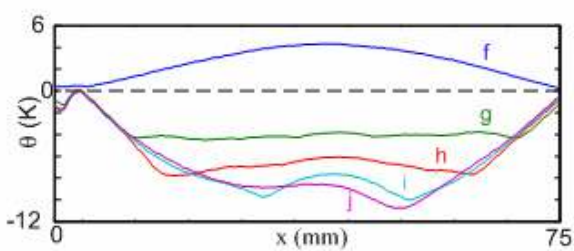

d)

Figure 6: Profiles evolution along one tube generating line during the tensile test performed on specimen 1. a) Axial strain profile $E_{x x}(x, y=0, t)$ during loading. b) Temperature variation $\theta(x, y=0, t)$ during loading. c) $E_{x x}(x, y=0, t)$ during unloading. $\left.d\right) \theta(x, y=0, t)$ during unloading.

Fig.7 represents, for specimen 2, the evolution of the Green-Lagrange axial strain profiles $E_{x x}(x, y=0, t)$ and the temperature variation $\theta(x, y=0, t)$ along one tube generating line at the selected times marked in Figure 4.b.

a)

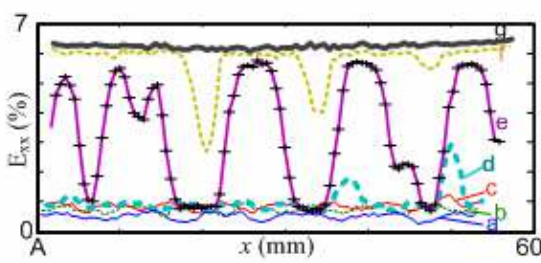

b)



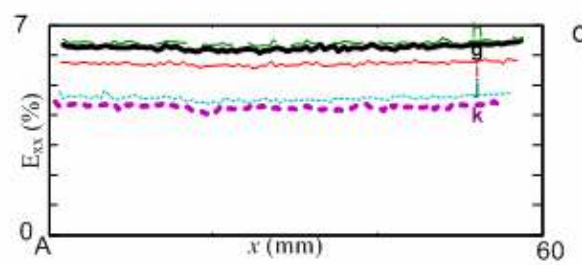

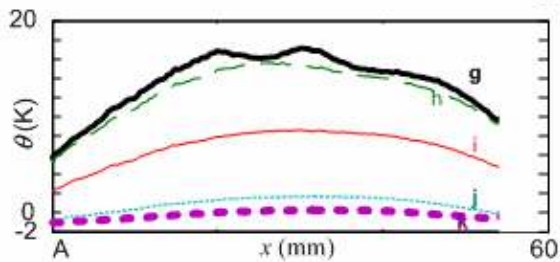

c)

d)

Figure 7: Profiles evolution along one tube generating line during the tensile test performed on specimen 2. a) Axial strain profile $E_{x x}(x, y=0, t)$ during loading. b) Temperature variation $\theta(x, y=0, t)$ during loading. c) $E_{x x}(x, y=0, t)$ during unloading. $\left.d\right) \theta(x, y=0, t)$ during unloading.

\section{Discussion}

Specimen 1 is fully superelastic at the testing temperature but the maximum strain during loading is $5.8 \%$ so that the unloading proceeds before the end of the loading stress plateau (stage 2 of Fig. 2). Since specimen 2 is initially austenite, A-R and R-M forward transformations are important deformation mechanisms during loading which is performed beyond the stress plateau. However, the testing temperature was not high enough to observe the reverse stress plateau during the unloading (stage 5 of Fig. 2).

The stages 1, 2 and 4 will be thus analysed for the two specimens, stage 3 only for the specimen 2 and stage 5 and 6 for the sample 1. Fig. 5 to 7 conjure up the following comments:

Stage 1 (O-b of Fig.4a and O-b of Fig.4b): During this stage, the deformation of the two samples proceeds homogeneously in the observed region, as demonstrated in the snapshot (a) of Fig. 5 and along the axial profiles of Fig. 6(a) and 7(a). An almost homogeneous temperature increase is observed for both specimens, starting as soon as the tensile deformation proceeds. It is also observed that both stress $\sigma_{0}$ and strain $\varepsilon$ but also temperature variation $\theta$ increase almost linearly with time. Such temperature increase shows that deformation mechanisms do not involve pure elasticity, which alone would induce a temperature decrease. 
Stage 2 (b-e of Fig.4a and c-f of Fig.4b): As soon as the stress peak appears, localisation phenomena are observed both in strain and temperature fields. In both tensile tests, the localizations take the shape of helical bands. The helical bands turn around the tube with a constant angle of approximately $59^{\circ}$ toward the vertical axis. Only one band is observed for specimen 1 whereas, on the second specimen, a second band starts at the end of the stress plateau (profile e in Figs 7.a). The end of the loading on specimen 1(time (e) Fig 4.a) is before the end of the stress plateau. At time (e) on sample 1, the strain profile is still strongly localized (Fig 6.a). Due to thermal conduction with the gripping system, the temperature field analysis is difficult.

Stage 3 (g-h of Fig.4b): The $\theta$ and $E_{x x}$ profiles of Fig. 7.c and 7.d show that the temperature is not homogeneous at time $(\mathrm{g})$ in spite of a homogeneous deformation of the tube. This is obviously due to the temperature history during the loading. It is worth noting that the almost homogeneous strain increase between times $(\mathrm{g})$ and $(\mathrm{h})$ is accompanied at any point of the tube by temperature decreases.

Stage 4 (e-f of Fig.4a and h-j of Fig.4b): The strain decreases homogeneously, even for the sample 1 which is deformed heterogeneously at time (e); this homogeneous strain decrease is accompanied by a strong temperature decrease, with smooth bell-shaped temperature profiles.

Stage 5 (g-i of Fig.4a): The strain decreases heterogeneously (times (f), (g) and (h) in Fig. 6.c). The temperature decreases below room temperature, as shown in Fig. 6.d.

Stage 6 (i-j of Fig.4a): The strain field is homogeneous again and the temperatures $\theta$ are heterogeneous and below ambient temperature.

The analysis of both strain and temperature variation fields reveal homogeneous and localised phenomena during tensile tests. The kinematic and morphology of localisation can be investigated. Since the measured temperature is only the consequence of internal heat sources and conduction phenomena with the gripping system and through the sample, the analysis of temperature remain qualitative and difficult. An estimation of the associated heat sources would provide precious quantitative information. Moreover it will give interesting data for future theoretical development concerning modelling of the thermomechanical behaviour of NiTi shape memory alloy.

So far, the nature of the cooling during stage 4 is unclear for both specimens 1 and 2. The next section will focus this stage 4 showing homogeneous strain decrease and try to answer this question by estimating homogeneous heat sources.

During the plateau (2 and 5) the morphology and kinematic of the localisation is obtained using the strain fields. As soon as the localisation starts, temperature localisations can be observed but are difficult to analysis after few seconds especially during the unloading. At the beginning of the unloading, the temperature field is strongly heterogeneous and the mean temperature is above room temperature. The last section will present a method to estimate $2 \mathrm{D}$ heat sources necessary in stage involving heterogeneous heat sources and temperature fields such as stage 2 and 5.

\section{From full-field measurements to the analysis of deformation mechanisms}

Heat conduction equations. Neglecting any heat source of external origin, heat transfers are ruled by the following local heat conduction equation [14-16]:

$$
C \dot{T}-\frac{k}{\rho} \operatorname{lap} T=\dot{q},
$$

where $T$ and $\dot{T}$ denote the temperature and its rate at any point of the body, respectively. The term "lapT" stands for the laplacian operator applied to the temperature field. It is assumed that the heat conduction is governed by the standard Fourier's law, with an isotropic, uniform and constant thermal conductivity $k$. The terms $\rho$ and $C$ denote respectively the mass density and the specific heat assumed both uniform and constant, $\dot{q}$ is the specific heat source rate (in $\mathrm{W} / \mathrm{kg}$ ). The experimental values for NiTi alloy of $C$ range from 450 to $600 \mathrm{~J} \mathrm{~kg}^{-1} \mathrm{~K}^{-1}$, and those for $\mathrm{k}$ from 8 to 
$28 \mathrm{~W} \mathrm{~m}^{-1} \mathrm{~K}^{-1}$ [17]. In this study, $C$ and $k$ were taken constant and equal to $490 \mathrm{~J} \mathrm{~kg}^{-1} \mathrm{~K}^{-1}$ and $15 \mathrm{~W} \mathrm{~m}^{-}$ ${ }^{1} \mathrm{~K}^{-1}$, respectively.

If the temperature field is homogeneous, the equation (1) can be written in a simpler form when heat sources $\dot{q}$ are homogeneously distributed in a specimen surrounded by ambient medium at temperature $T_{0}$ and when specimen temperatures are not too far from the thermal equilibrium [15]. At any point of the outer surface of the tube, this simplified model reads :

$C\left(\frac{\partial \theta}{\partial t}+\frac{\theta}{\tau_{\mathrm{eq}}}\right)=\dot{q}$,

where $\theta=T-T_{0}$ is the temperature variation. The parameter $\tau_{\mathrm{eq}}$ represents a characteristic time reflecting heat losses both by convection through inner and outer surfaces of the tube and by conduction towards the grip's zones. More detail on the estimation of $\tau_{\mathrm{eq}}$ and the hypothesis are presented in [18].

Hypotheses for the deformation mechanisms. It is well known that deformation mechanisms for NiTi shape memory alloys include elastic distortion of the atomic lattice and additional mechanisms associated with martensitic and $\mathrm{R}$ phase(s) transformation(s) and variants (de)twinning [19]. Local plastic accommodation of the transformation(s) may also be involved but is effective only for large local strain [7]. The heat source rate $\dot{q}$ involved in (2) is thus divided into a first rate $\dot{q}_{t r}$ due to phase(s) transformation(s), a second one $\dot{q}_{t h e l}$ due to the usual thermoelastic coupling and a last one $\dot{q}_{d i s s}$ due to intrinsic dissipation induced by irreversible phenomena like plasticity and frictional barriers opposing interfacial motion :

$\dot{q}=\dot{q}_{t r}+\dot{q}_{t h e l}+\dot{q}_{\text {diss }}$ with $\dot{q}_{\text {thel }}=-\frac{\alpha T_{0} \dot{\sigma}_{0}}{\rho}$,

where $\alpha$ is the thermal expansion coefficient. The classical additive decomposition of the strain rate $\dot{\varepsilon}$ is assumed :

$\dot{\varepsilon}=\dot{\varepsilon}_{e l}+\dot{\varepsilon}_{i n} \quad$ with $\quad \dot{\varepsilon}_{e l}=\frac{\dot{\sigma}_{0}}{\mathrm{E}}$,

where $\dot{\varepsilon}_{e l}$ and $\dot{\varepsilon}_{i n}$ are the elastic and inelastic strain rates, respectively. The inelastic strain rate includes the rate due to transformation(s) $\dot{\varepsilon}_{t r}$ and the rate due to variants (de)twinning $\dot{\varepsilon}_{d e}$. In the present analysis, the rate due to plasticity is neglected, together with $\dot{q}_{d i s s}=0$.

Lastly, for any phase transformation, both strain rate $\dot{\varepsilon}_{t r}$ and heat source rate $\dot{q}_{t r}$ will be assumed proportional to the transformation fraction rate $\dot{f}[16,20]$ :

$\dot{\varepsilon}_{t r}=\dot{f} \varepsilon_{t r} \quad$ and $\quad \dot{q}_{t r}=\dot{f} \Delta H_{t r}$,

where $\varepsilon_{t r}$ and $\Delta H_{t r}$ stand for the transformation strain and specific enthalpy for a complete transformation $(f=1)$.

Deformation mechanisms during homogeneous unloading stage. Equations developed above are now used in order to further analyse the beginning of the unloading of the sample 2 (stage 4 conventionally attributed to elastic deformation and detwinning of the oriented $\mathrm{M}$ variants formed during loading). In this stage, temperature variation $\theta$, stress $\sigma_{0}$ and strain $\varepsilon$ decrease almost linearly with time (Fig 8.a). Due to the decrease of temperature (endothermic heat source), three main mechanisms are involved during the unloading, i.e. elasticity, detwinning and reverse transformation, plasticity being neglected. Taking into account the equations (4), the additive decomposition of the strain rate is thus expressed as :

$\dot{\varepsilon}=\dot{\varepsilon}_{e l}+\dot{\varepsilon}_{t r}+\dot{\varepsilon}_{d e}=\frac{\dot{\sigma}_{0}}{\mathrm{E}}+\frac{\dot{q}_{\mathrm{tr}}}{\Delta \mathrm{H}_{\mathrm{tr}}} \varepsilon_{\mathrm{tr}}+\dot{\varepsilon}_{d e}$ 
From these measurements and the equation (6), the absolute values of the elastic $\dot{\varepsilon}_{e l}$, reverse transformation $\dot{\varepsilon}_{t r}$ and detwinning $\dot{\varepsilon}_{d e}$ strain rates are plotted in Fig.8.b as functions of the strain $\varepsilon_{l 0}$ between times $(h)$ and $(j)$.
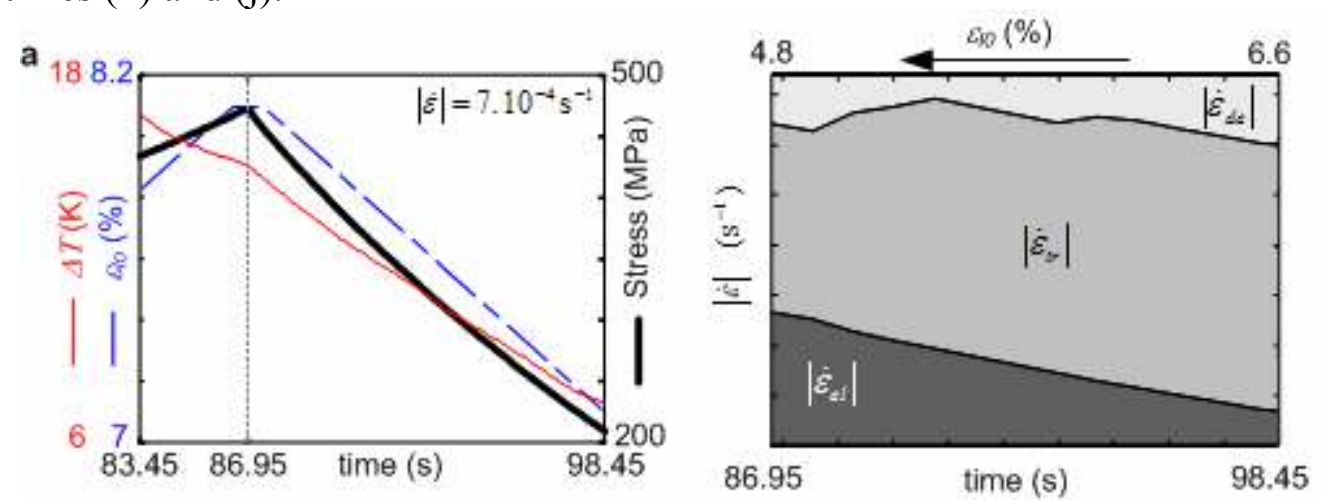

b

Figure 8: (a) Stress $\sigma_{0}$ (thick curve), strains $\varepsilon$ (dashed curve) and temperature variations (thin curve) as functions of time during stage 4 of sample 2. (b) Estimation of the evolution of the elastic, transformation and detwinning strain rates.

As evident from fig.8.b, deformation mechanisms other than pure elastic distortion of crystalline lattice occur as soon as the stress starts to decrease. From the above rough analysis, it is suggested that reverse phase transformation has a predominant effect during the whole unloading. Its contribution to the overall strain rate is almost constant and equal to half the total strain rate. Decreasing the strain $\varepsilon$ leads to reduce the elasticity contribution and to increase the detwinning phenomena. It is worth concluding that these results deny the usual assumption of elastic unloading which is commonly made in thermomechanical modelling for stage 4 of Fig. 1.

Heat source estimation during heterogenous stages. It is very difficult to analyse quantitatively the observed heterogeneous deformation mechanisms from temperature maps such as that ones shown in Fig. 3. Instead, the determination of local heat source fields are much more appropriated, to detect and analyse the phase transformations.

The local heat sources can be estimated from the heterogeneous temperature field by solving the heat source equation (1). Unfortunately, the IR camera allows measuring only temperatures at the outer surface of the considered tubes, so that further assumptions are required to circumvent this problem. The following sets of hypotheses are stated, as previously achieved in the case of thin flat samples $[15,21]$ : the initial temperature of the tube $T_{0}$ is supposed to be homogeneous and equal to the ambient air temperature $T_{a}$ assumed constant during the whole tensile test; the thickness of the tube $e$ is small compared to its mean radius $R$ and the temperature and heat sources are assumed to be homogeneous within the thickness.

By integrating equation (1) along the thickness of the tube, it is now possible to obtain an estimation of the averaged heat sources $\left\langle s_{i}\right\rangle$, approximating the surface temperature given by the IR camera to the averaged temperature variation $\langle\theta\rangle=\frac{1}{e} \int_{R-e / 2}^{R-e / 2}\left(T-T_{0}\right) d r$.

$\rho C\left(\langle\theta\rangle_{, t}+\frac{\langle\theta\rangle}{\tau_{t h}}-\right.$ dlap $\left._{2}\langle\theta\rangle\right)=\left\langle s_{i}\right\rangle$,

where $d=k /(\rho C)$ is the thermal diffusivity and $\tau_{t h}=\rho C / h_{e q}$ represents the characteristic time of the radial heat loss by convection and radiation, $\langle\theta\rangle_{, t}$ represent the time derivative of the averaged temperature variation field, $\operatorname{lap}_{2}\langle\theta\rangle$ the laplacian. 
The process to estimate the heat sources fields is based on the left hand side estimation of the equation (7) by using the experimental temperature variation fields $\langle\theta\rangle$ ([15]). These fields are discrete and noisy data, obtained at the outer surface $(r=R+e / 2)$ of the tube. From the noisy and discrete temperature field, time derivative and laplacien are then estimated in cylindrical coordinates using image processing filter and discrete derivation operators, in time and space ([14]). The image filtering, necessary to estimate operators on the noisy data, is a delicate operation and is obtained by low pass filters applied by Fast Fourier Transform.

\section{Example of local heat sources estimation at two characteristics times on sample 1.}

The processing method was applied on the temperature fields of specimen 1 . The comparison of temperature field and heat sources field at time (d) of Fig.4.a are plotted in Figure 9. $a$ and $b$ respectively. At this time (middle of the stress plateau), the temperature variation field was about 7 $\mathrm{K}$ above room temperature and strongly heterogeneous (Profile (d) in Fig.6.b). This temperature variation field was hard to interpret mostly due to strong conduction phenomena. The heat source field in Fig.9.b shows several peaks that are with a similar angle than the strain field discontinuities (Figure 5). Three strong peaks were close to the grips with a magnitude of 4 to $610^{6} \mathrm{~W} / \mathrm{m}^{3}$. In the middle of the sample two sets of parallel fronts close to each other with a smaller amplitude (1 to 2 $10^{6} \mathrm{~W} / \mathrm{m}^{3}$ ) are observed. Those fronts were moving away from each other. Outside of theses peaks, the heat sources were strongly reduced.
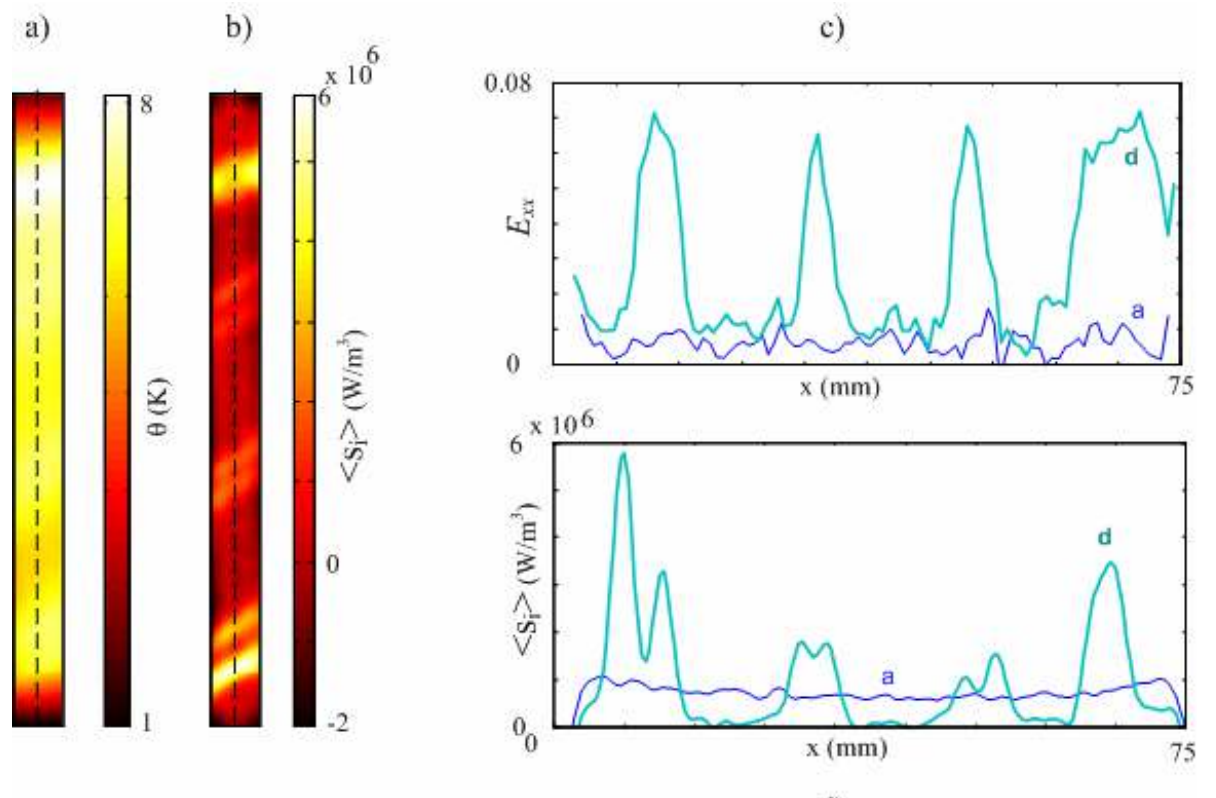

d)

Figure 9 : Tensile test on sample 1. a) Temperature variation field $\theta(M, t)$ at time (d) of Fig.4.a. b) Heat sources $\left\langle s_{i}(M, t)>\right.$ estimated at the same time. $\left.c\right)$ Axial strain profiles along a generating line of the tube $E_{x x}(x, y=0, t)$ at times $(a)$ and (d) of Fig.4.a. b) Heat sources $<s_{i}(x, y=0, t)>$ profiles along the same generating line at times $(a)$ and $(d)$.

The heat source profiles along a generating line of the sample are compared to the axial strain profiles in Figure 9.d and c at times (a) and (d) respectively. In the homogeneous stage 1 (time a), both heat source and strain profiles were homogeneous. The heat sources were exothermic estimated of the order of $10^{6} \mathrm{~W} / \mathrm{m}^{3}$. During the stress plateau (stage 2, time d), the strain field was strongly localised. The helical band initiated on the top grip has reached the bottom one; four peaks were present along the sample. Although, the temperature variation are hard to analyse (cf Figure 6.b, time (d)), the heat source profile shows clearly peaks and discontinuities at the same position as the strain discontinuities. The amplitudes of the discontinuities give information on the kinetics of 
the phase transformations. The fronts propagate faster where the heat sources are higher. When no strain variation was observed, the heat sources were weak. Inside the highly deformed area, the heat sources decreased strongly, revealing a reduction of the phase transformation kinetic.

\section{Conclusions}

Although experimental observations of tensile tests on NiTi SMA and of localisation phenomena occurring during these tests can be found in the references cited earlier [1-9, 22], simultaneous measurements of temperature and strain fields are unique. They provide new results enabling to revisit the conventional understanding of homogeneous and heterogeneous phenomena occurring during tensile tests on NiTi SMA.

These simultaneous measurements, have allowed us to observe both homogeneous and localized stages during the tensile tests. The strain field is especially useful to analyze the kinetic of localized stage. Temperature field only gives qualitative information. An estimation of local heat source can be obtained from the temperature field. Depending of the observed phenomena, two models have been presented: A 1D thermal estimation suitable for homogeneous stage has been proposed, and a more complex, 2D model resolution when the temperature field are localised. Such heat source estimation enables to better analyse the deformation mechanism.

\section{References}

[1] Miyazaki, S., Imai, T., Otsuka, K. and Suzuki, Y, Scripta Metall., 1981, 15, 853-56.

[2] Shaw, J.A. and Kyriakides, S., J. Mech. Phys. Solids, 1995, 43, 1243-81.

[3] Shaw, J.A. and Kyriakides S., Acta Mater., 1997, 45, 683-700.

[4] Favier, D., Liu, Y., Orgéas, L. and Rio ,G., 2001, in Sun Q.P. (Ed), Solid Mechanics and Its Applications, 101, Kluwer Ac. Pub., New-York, 205-212.

[5] Orgéas, L. and Favier, D., Acta Mater. , 1998, 46, 5579-91.

[6] Sun, Q.P. and Li, Z.Q., Int. J. Solids Struct., 2002, 39, 3797-809.

[7] Brinson, L.C., Schmidt, I. and Lammering, R., J. Mech. Phys. Solids, 2004, 52, 1549-1571

[8] Ng, K.L. and Sun, Q.P., Mech. Mater., 2006, 38, 41-56.

[9] Gadaj, S.P., Nowacki, W.K. and Pieczyska, E.A., Infrared Phys. Techn., 2002, 43, 151-155.

[10] Liu, Y., Liu, Y. and Van Humbeeck, J., Scripta Mater., 1998, 39, 1047-55.

[11] Chache, M. PhD Dissertation, Université Joseph Fourier Grenoble I, 2004.

[12] Vacher, P., Dumoulin, S., Morestin, F. and Mgil-Touchal, S., Proc. of Instn Mech Engrs, ImechE, 1999, 213C, 811-17.

[13] Louche, H., Bouabdallah, K., Vacher, P., Coudert, T., Balland, P., exp Mech, 2008, online DOI-10.1007/s11340-008-9125-5

[14] Schlosser, P., Louche, H., Favier, D. and Orgéas, L., Strain, 2007, 43, 260-271

[15] Chrysochoos, A. and Louche, H., Int. J. Eng. Sci., 2000, 38, 1759-88.

[16] McCormick, P.G., Liu, Y. and Miyazaki, S., Mater. Sci. Eng., 1993, A167, 51-56.

[17]Faulkner, M. G., Amalraj, J. J. and Bhattacharyya, A. Smart Mater. Struct. 2000, 9, 632-639.

[18] Favier, D., Schlosser, P., Louche, H., Orgéas, L., Vacher, P. Debove, L., Acta Mater. 2007, 55, 5310-5322

[19] Sittner, P., Novak, V., Lukas, P. and Landa M., J.Eng. Mat. Tec., ASME, 2006, 128, 268-278.

[20] Lexcellent, C. and Vacher, P., Arch. Mech., 1993, 45 1, 135-155.

[21] Louche, H. PhD Dissertation, Montpellier University, 1999.

[22] S. Daly, G. Ravichandran and K. Bhattacharya, Acta Materialia 2007, 55, 3593-3600. 